\title{
Synovial chondromatosis of the temporomandibular joint: a case report
}

\author{
O Barraclough, G Wilson, A Power
}

Bradford Teaching Hospitals NHS Foundation Trust, UK

\section{ABSTRACT}

Synovial chondromatosis is a rare benign condition. It most commonly affects the large joints. Presentation in the temporomandibular joint is rare.

Our case was an incidental radiological finding and not diagnosed immediately, highlighting the ease with which conditions such as this can be missed, particularly in asymptomatic patients. Only 45\% of patients with synovial chondromatosis show radiographic changes. Findings as significant as ours are unusual. An increased professional awareness of the radiological signs of synovial chondromatosis would be beneficial to improve diagnosis and prognosis for patients.

\section{KEYWORDS}

Synovial chondromatosis - Temporomandibular joint - Osteochondromatosis

Accepted 23 April 2020

CORRESPONDENCE TO

Olivia Barraclough, E: olivia.barraclough1@nhs.net

\section{Introduction}

Synovial chondromatosis is characterised by metaplasia of the synovial membrane, which proliferates to produce cartilaginous nodules. Clinical symptoms of synovial chondromatosis affecting the temporomandibular joint include swelling, pain, crepitation, malocclusion and joint dysfunction. ${ }^{1}$ These symptoms may mimic those of other nonspecific temporomandibular joint diseases, and therefore diagnosis is often delayed. We present an unusual case where initial diagnosis occurred before the onset of any symptoms. The aim of this report is to highlight the low incidence of synovial chondromatosis affecting the temporomandibular joint and the importance of consistent, methodical radiograph analysis.

\section{Case report}

Our 32-year-old male patient attended the maxillofacial department following routine referral for wisdom tooth extraction. An orthopantomogram showed a speckled radiolucency superimposed over the temporomandibular joint/temporal region, but this was overlooked on initial assessment (Fig 1). With only $45 \%$ of cases of synovial chondromatosis showing radiographic changes, findings as significant as ours are particularly unusual. ${ }^{2}$ Clinical examination revealed a corresponding firm bony lump in this region, with no other signs or symptoms. The appearance on a mandible computed tomography scan (Fig 2) reflected primary synovial osteochondromatosis. Radiology advised that no surgical intervention was necessary. After discussion with the patient, it was agreed to proceed with conservative management only.

The patient subsequently returned for review, reporting intermittent pain on the left side, particularly when chewing. The discomfort was worse at night, with associated jaw stiffness. On examination, the patient displayed no restriction of movement. He was offered open temporomandibular joint surgery. The risks were discussed, including those of facial weakness, scarring, limitation to the jaw movement, and a requirement of further surgical input.

Using a preauricular surgical technique, dissection to the root of the zygoma followed by periosteal elevation, the capsule was located (Fig 3). On opening the capsule, multiple chondromata were discovered, as anticipated. These were removed to reveal a healthy disc with no evidence of perforation.

Aftercare instructions and physiotherapy exercises were recommended to ensure resolution of normal mouth opening and reduce the risk of ankylosis of the temporomandibular joint. Immediately postoperatively, the patient demonstrated no alteration in hearing, satisfactory mouth opening and normal facial nerve function. There was some mild paraesthesia to the left temporal region postoperatively. This remained the same at three-week review.

Histopathology confirmed the diagnosis of synovial chondromatosis, showing multiple nodules of cartilage (combined maximum dimension $50 \mathrm{~mm}$ ) and focal bone formation. 


\section{Discussion}

Cartilaginous metaplasia of synovial tissue leads to the deposition of cartilaginous nodules in the synovial membrane of the affected joint. ${ }^{3}$ These nodules have the potential to grow, calcify and ossify within the articular space and eventually detach as loose bodies within the joint. $^{2}$ These loose bodies can damage the smooth articular cartilage covering the joint, causing osteoarthritis. Prompt diagnosis is, therefore, imperative. Synovial chondromatosis may occur secondary to pre-existing joint diseases such as arthritis, trauma or infection, or exist as a primary condition. $^{3}$

Radiological images are an integral part of distinguishing synovial chondromatosis from other similar conditions, with pathohistological analysis mandatory in confirming the disease. ${ }^{1}$

Histologically, synovial chondromatosis is described in three stages of development. Primary synovial chondromatosis involves metaplasia in the synovial membrane without loose bodies. Secondary synovial chondromatosis shows

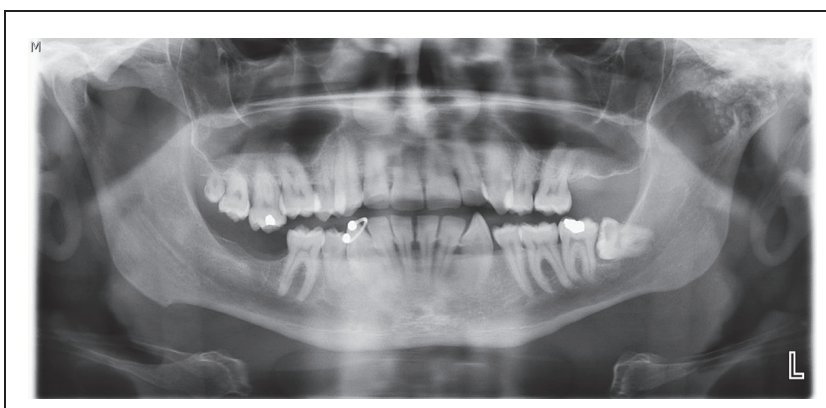

Figure 1 Orthopantomogram showing speckled radiolucency superimposed over the temporomandibular joint/temporal region

progressive metaplasia and detachment of loose bodies; these contain active chondrocytes partially surrounded by a synovial membrane. In the final stage, there is no longer any metaplastic activity in the region of the synovial membrane, and only detached bodies are found. ${ }^{4}$

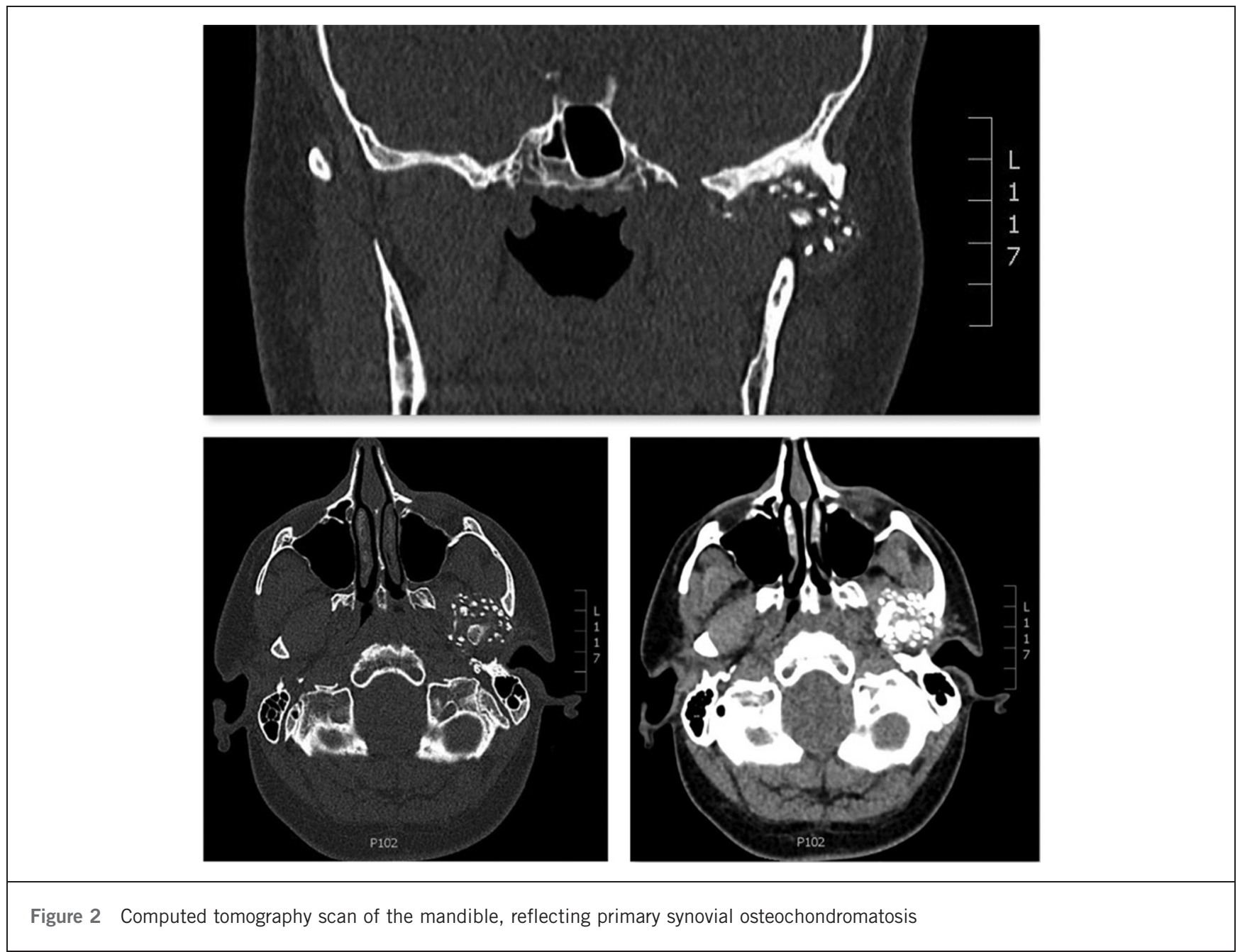




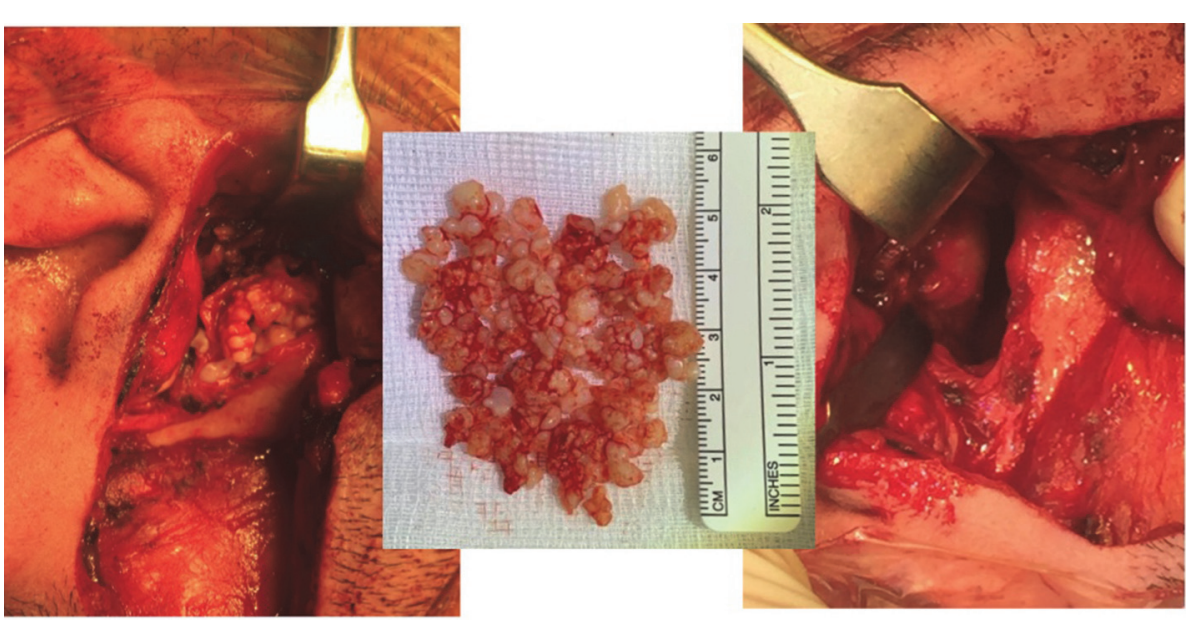

Figure 3 Dissection to the root of the zygoma followed by periosteal elevation revealed multiple chondromata

Synovial chondromatosis does not respond to non-surgical treatment or show spontaneous resolution. ${ }^{5}$ Arthroscopy is the least invasive technique, but it is difficult to retrieve the intraarticular loose bodies. This may be successful where the nodules are confined to a single joint compartment, do not exhibit extraarticular extension and the particle size is less than $3 \mathrm{~mm} .^{1}$

Open joint surgery to remove all the loose cartilaginous bodies and synovial membrane is usually sufficient to control disease in the majority of cases, but condylectomy and discectomy may also be considered. ${ }^{3}$

Dental professionals have a unique window of opportunity to screen for many conditions and diseases in the head and neck region. This can range from superficial skin lesions such as basal cell carcinoma, to deep conditions and bony pathology such as fibrous dysplasia. It is imperative that all special investigations are reviewed, reported and documented. Our case study demonstrates how pathology can be easily overlooked when a methodical clinical and radiographic assessment is not performed.

\section{Conclusion}

In summary, synovial chondromatosis does not commonly affect the temporomandibular joint. The majority of cases reported have been detected in symptomatic patients. As our case was an incidental finding, it highlights the importance of thorough analysis of all radiographs and the ease at which conditions such as this can be overlooked.

\section{References}

1. Ivask O, Leibur E. Synovial chondromatosis in the temporomandibular joint: case report with literature review. Baltic Dental Maxillofacial J 2015; 17: 97-101

2. Pinto A, Costa R, Chagas $S$ et al. Synovial chondromatosis of the temporomandibular joint successfully treated by surgery. Head Neck Pathol 2015; 9: 525-529

3. Singh K, Ghosh S, Verma M et al. Imaging findings in a case of synovial chondromatosis of temporomandibular joint. J Indian Acad Oral Med Radiol 2018; 30: 73-77

4. Koyama J, Ito J, Hayashi J et al. Synovial chondromatosis in the temporomandibular joint complicated by displacement and calcification of the articular disk: report of two cases. Am J Neuroradiol 2001; 22: 1203-1206

5. Lim S, Jeon SJ, Choi SS, et al. Synovial chondromatosis in the temporomandibular joint: a case with typical imaging features and pathological findings. Br J Radiol 2011; 84: e215-e218 